\title{
Empirical Equation for the Gravitational Constant with a Reasonable Temperature
}

\author{
Tomofumi Miyashita \\ Miyashita Clinic, Osaka, Japan \\ Email: tom_miya@plala.or.jp
}

How to cite this paper: Miyashita, T. (2020) Empirical Equation for the Gravitational Constant with a Reasonable Temperature. Journal of Modern Physics, 11, 1180-1192.

https://doi.org/10.4236/jmp.2020.118074

Received: July 14, 2020

Accepted: August 9, 2020

Published: August 12, 2020

Copyright $\odot 2020$ by author(s) and Scientific Research Publishing Inc. This work is licensed under the Creative Commons Attribution International License (CC BY 4.0).

http://creativecommons.org/licenses/by/4.0/

\begin{abstract}
Ted Jacobson discovered that gravity was related to thermodynamics. However, the calculated temperature using the Boltzmann area entropy is still not reasonable. We searched and discovered an empirical equation for the gravitational constant with a reasonable temperature. The calculated value was $3.20 \mathrm{~K}$, which is similar to the temperature of the cosmic microwave background of $2.73 \mathrm{~K}$. Then, we examined Yasuo Katayama's theory. For this purpose, we introduced the modified Wagner's equation, which is compatible with Jarzynski equality. Finally, using Ted Jacobson's theory, we proposed our theory of gravity with the Gibbs volume entropy.
\end{abstract}

\section{Keywords}

Gravitational Constant, Wagner's Equation, Jarzynski Equality, Ted Jacobson's Theory

\section{Introduction}

Ted Jacobson attempted to relate gravity and thermodynamics [1]. Subsequently, the theory of entropic gravity [2] was studied by Erik Verlinde. However, the calculated temperature values obtained are still not reasonable.

There are two kinds of entropy. The first is the Boltzmann area entropy, and the second is the Gibbs volume entropy ( $\left.S_{\text {Gibbs }}\right)$. The theory of entropic gravity is based on the Boltzmann area entropy. The temperature calculated using this approach was very small and not reasonable. According to Jarzynski, the Gibbs volume entropy $\left(S_{\text {Gibbs }}\right)$ is much stronger than the Boltzmann area entropy [3]. Therefore, instead of using the Boltzmann area entropy, we tried to use the Gibbs volume entropy to explain our empirical equation.

In the area of solid-state ionics, the open circuit voltage is governed by Wagn- 
er's equation. However, we noticed that during ion hopping, the chemical potential of ions should increase, and the electrical potential energy of ions should decrease. Both the values of the increase in the chemical potential and the decrease in the electrical potential energy are equal to the ionic activation energy. We noticed that the ionic activation energy can be defined as the solvation Hamiltonian mean force $(\phi)$ [4]. The solvation Hamiltonian mean force $(\phi)$ in the electrolytes can be defined from the separation of the Boltzmann distribution. Furthermore, the solvation Hamiltonian mean force $(\phi)$ can be measured experimentally.

In our theory, the energy of the rest mass slightly decreases after pair production. At the same time, the Gibbs volume entropy $\left(S_{\text {Gibbs }}\right)$ should slightly increase. Both the values of the increase and the decrease are equal to the solvation Hamiltonian mean force $(\phi)$. However, the ratio between the solvation Hamiltonian mean force $(\phi)$ and the energy of the rest mass are too small and cannot be observed. We assumed that the solvation Hamiltonian mean force $(\phi)$ should increase with increasing distances. Then, the rest mass should increase with increasing distances. The Gibbs volume entropy $\left(S_{\text {Gibss }}\right)$ should increase with increasing distances.

The rest of the paper is organized as follows: in Section 2, we present our empirical equations and our conclusions. In Section 3, we attempt to explain our empirical equations using the modified Wagner's equation. In Section 4, using Ted Jacobson's method, we showed the obtained conclusions for our theory of gravity.

The schematic view of our project is shown in Figure 1.

The central concept from our experimental results is related to Wagner's equation in electrochemistry.

\section{Empirical Equation for the Gravitational Constant}

\subsection{Our Empirical Equation}

Our empirical equation is (quoted from Wikipedia

https://en.wikipedia.org/wiki/Proton)

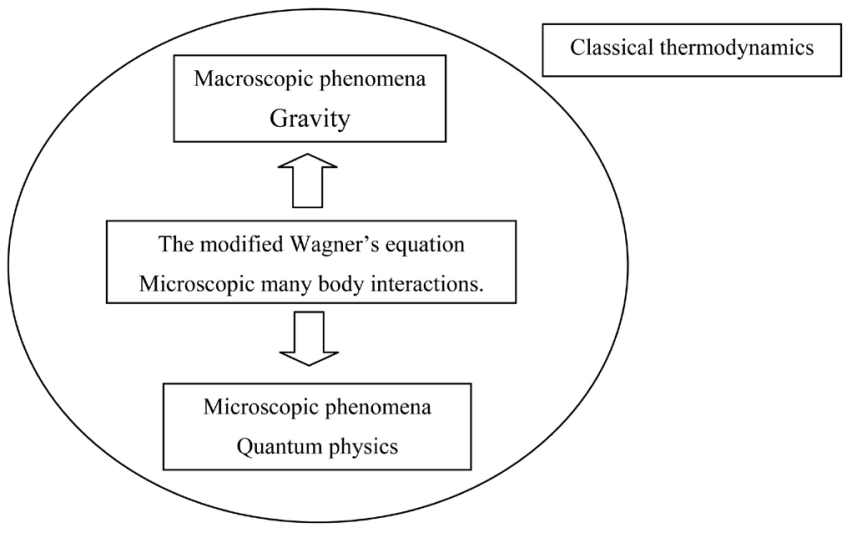

Figure 1. Schematic view of our project. 


$$
\frac{G m_{p}}{r_{p}} \times 1 \mathrm{~kg}=3 \mathrm{kT}
$$

$G$ : gravitational constant, $6.6743 \times 10^{-11}\left(\mathrm{~m}^{3} \cdot \mathrm{kg}^{-1} \cdot \mathrm{s}^{-2}\right)$.

$m_{p}$ : the rest mass of a proton, $1.67 \times 10^{-27}(\mathrm{~kg})$.

$r_{p}$ : charge radius, $8.41 \times 10^{-16}(\mathrm{~m})$.

$k$. Boltzmann constant, $1.38 \times 10^{-23}(\mathrm{~J} / \mathrm{K})$.

T: temperature $(\mathrm{K})$.

$1 \mathrm{~kg}$ : the standard mass $(\mathrm{kg})$.

The temperature $(T)$ calculated using this formula was $3.20 \mathrm{~K}$, which is similar to the temperature of the cosmic microwave background of $2.73 \mathrm{~K}$. The standard mass ( $1 \mathrm{~kg}$ ) must be used due to the definition of the gravitational constant. This problem was explained in Section 3.1.

\subsection{Our Conclusions from Our Empirical Equation}

Equations (2)-(4) are our conclusions.

$$
\begin{gathered}
\phi=3 k T . \\
m_{p} c^{2}=h v-3 k T \\
\frac{\mathrm{d} S_{\text {Gibbs }}}{\mathrm{d} r} T=\frac{3 n k T}{r}
\end{gathered}
$$

where $h$ and $v$ are the Planck constant and the frequency, respectively. $n$ is the number of protons, and $r$ is the distance from a large mass $\left(n \times m_{p}\right)$.

\section{Explanation for Our Empirical Equation}

\subsection{Consideration of the Law of Universal Gravitation}

This section is strongly influenced by Yasuo Katayama [5].

http://home.catv.ne.jp/dd/pub/pot/pot.html\#0.

He thought that, according to Newton, the energy can be obtained infinitely from the gravitational potential energy, but according to Einstein, there should be an upper limit $\left(m c^{2}\right)$. Thus, the rest mass should decrease with decreasing the gravitational potential. Equation (16) was quoted from Yasuo Katayama.

The gravitational potential $(\Phi(r))$ is given by

$$
\Phi(r)=-\frac{G M}{r},
$$

where $M$ and $r$ are the mass and the distance from the mass, respectively. Then, the work $(W)$ is given by

$$
W=m \times\left(\Phi\left(r_{2}\right)-\Phi\left(r_{1}\right)\right)=m \times\left(\frac{G M}{r_{1}}-\frac{G M}{r_{2}}\right)
$$

where $m$ is a sufficiently small mass. $r_{1}$ and $r_{2}\left(>r_{1}\right)$ are the distances from mass $M$. Therefore,

$$
W=\int_{r_{1}}^{r_{2}} m \times \frac{\mathrm{d} \Phi(r)}{\mathrm{d} r} \mathrm{~d} r
$$


Then,

$$
\begin{gathered}
\frac{\mathrm{d} W}{\mathrm{~d} r}=m g \\
g=\frac{\mathrm{d} \Phi(r)}{\mathrm{d} r}
\end{gathered}
$$

Next, from the mass-energy equivalence and the Law of the Conservation of Energy, we obtain

$$
m_{2} c^{2}=m_{1} c^{2}+W
$$

Therefore,

$$
m_{2}-m_{1}=\frac{W}{c^{2}}
$$

$m$ should increase with the increasing of $r$.

$$
\frac{\mathrm{d} m}{\mathrm{~d} r}=\frac{1}{c^{2}} \frac{\mathrm{d} W}{\mathrm{~d} r} .
$$

From Equations (5) and (9), we obtain

$$
\frac{\mathrm{d} m}{\mathrm{~d} r}=\frac{1}{c^{2}} m g .
$$

Therefore, from Equation (6) and Equation (10), we find that

$$
\frac{\mathrm{d} m}{\mathrm{~d} r}=\frac{1}{c^{2}} m \frac{\mathrm{d} \Phi(r)}{\mathrm{d} r} .
$$

Then,

$$
\mathrm{d} m=\frac{1}{c^{2}} m \mathrm{~d} \Phi(r) .
$$

Then, we assumed that $m$ should be the minimum mass $\left(m_{p 1}\right)$ when $r$ is the minimum distance $\left(r_{p 1}\right)$.

$$
\ln \left(\frac{m_{p 2}}{m_{p 1}}\right)=\frac{1}{c^{2}}\left(\Phi\left(r_{p 2}\right)-\Phi\left(r_{p 1}\right)\right)
$$

Thus,

$$
\ln \left(\frac{m_{p 2}}{m_{p 1}}\right)=\frac{G}{c^{2}}\left(\frac{M_{1}}{r_{p 1}}-\frac{M_{2}}{r_{p 2}}\right)
$$

When $M$ is $n$ (the number of protons) $\times m_{p}$,

$$
\begin{aligned}
M_{1} & =n \times m_{p 1} \\
M_{2} & =n \times m_{p 2}
\end{aligned}
$$

From Equations (17)-(19),

$$
\ln \left(\frac{m_{p 2}}{m_{p 1}}\right)=\frac{n G}{c^{2}} \times\left(\frac{m_{p 1}}{r_{p 1}}-\frac{m_{p 2}}{r_{p 2}}\right)
$$

when $r_{p 2}$ is infinitely large. 


$$
\ln \left(\frac{m_{p 2}}{m_{p 1}}\right)=\frac{n G}{c^{2}} \times \frac{m_{p 1}}{r_{p 1}}
$$

Therefore,

$$
\frac{c^{2}}{n} \ln \left(\frac{m_{p 2}}{m_{p 1}}\right)=G \times \frac{m_{p 1}}{r_{p 1}}
$$

When $M_{1}$ is $1 \mathrm{~kg}, n$ is $6.02 \times 10^{26}\left(=1000 \times 10^{23}\right)$. The value of $n$ is calculated from Avogadro's number.

$$
1 \mathrm{~kg}=n \times m_{p 1}
$$

thus forming Equation (22) and Equation (23),

$$
m_{p 1} c^{2} \times \ln \left(\frac{m_{p 2}}{m_{p 1}}\right)=1 \mathrm{~kg} \times G \times \frac{m_{p 1}}{r_{p 1}}
$$

Then, $n$ disappeared. This does not mean that $n$ is unimportant. As shown in Equation (20), $n$ is clearly meaningful. We tried to explain the influence of 1 proton on the gravitational potential, and then, $n$ disappeared. When Newton defined $10 \mathrm{~kg}$ as the standard mass, the value of $G$ should change. However, Equation (24) should not change. The real meaning of Equation (24) was discussed in the later section.

\subsection{Consideration from Wagner's Equation}

This section is the most important and is quoted from our own experimental results [4]. In this section, we tried to explain the concept of the solvation Hamiltonian mean force from the perspective of thermodynamics in relation to hopping conduction.

A solid oxide fuel cell (SOFC) directly converts the chemical energy of a fuel gas, such as hydrogen or methane, to electrical energy. A solid oxide film is used as the electrolyte, where the main carriers are oxygen ions. As electrolytes, YSZ and SDC (samaria-doped ceria) are well known. The open circuit voltage (OCV) is calculated from Wagner's equation.

$$
J_{\mathrm{O}_{2}}=-\frac{R T}{16 F^{2} L} \int_{\ln \left(p O_{2}^{\text {anode }}\right)}^{\ln \left(p O_{2}^{\text {cathode }}\right)} \frac{\sigma_{\mathrm{el}} \sigma_{\text {ion }}}{\sigma_{\mathrm{el}}+\sigma_{\text {ion }}} \mathrm{d} \ln \left(p O_{2}\right)
$$

where $J_{\mathrm{O}_{2}}$ and $\mathrm{pO}_{2}$ are the $\mathrm{O}_{2}$ flux and the $\mathrm{O}_{2}$ partial pressure, respectively; $\mathrm{pO}_{2}^{\text {cathode }}$ and $\mathrm{pO}_{2}^{\text {anode }}$ are the $\mathrm{O}_{2}$ partial pressures at the cathode and the anode, respectively; $R, T$, and $F$ are the gas constant, the absolute temperature, and Faraday's constant, respectively; $L$ is the thickness of the membrane or film; and $\sigma_{\mathrm{el}}$ and $\sigma_{\mathrm{ion}}$ are the conductivities of the electrons and oxygen vacancies, respectively.

However, we have previously reported two experimental problems related to Wagner's equation.

Problem 1: The unchanged OCV during electrode degradation [6] [7].

Problem 2: The equilibration process in response to a change in the anode gas 
using thick SDC electrolytes [8].

To solve these problems, we proposed the concept of the solvation Hamiltonian of mean force $(\phi)$, which was discussed by Jarzynski theoretically [3]. After introducing the concept of the solvation Hamiltonian of mean force $(\phi)$, we noticed that our model was still compatible with Wagner's equation. Thus, we called our model "the modified Wagner's equation".

Using only three graphs, the solvation Hamiltonian of mean force $(\phi)$ can be explained. The Boltzmann distribution of oxygen ions in the electrolyte at 1073 $\mathrm{K}$ is displayed in Figure 2. The ions with energies exceeding the ionic activation energy become carriers (hopping ions). Figure 3 presents an incorrect carrier distribution. According to Jarzynski, an accurate distribution should be a canonical ensemble, as shown in Figure 4. The solvation Hamiltonian of mean force $(\phi)$ can be defined and is illustrated in Figure 3.

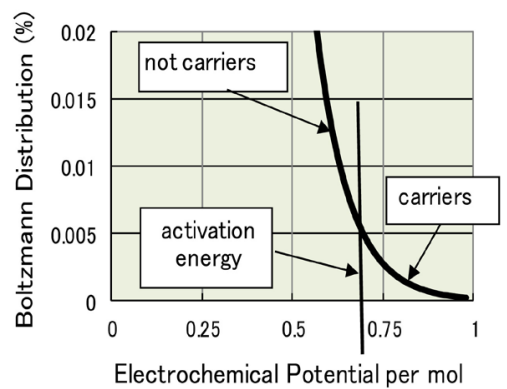

$(\mathrm{eV})$

Figure 2. Boltzmann distribution at $1073 \mathrm{~K}$.

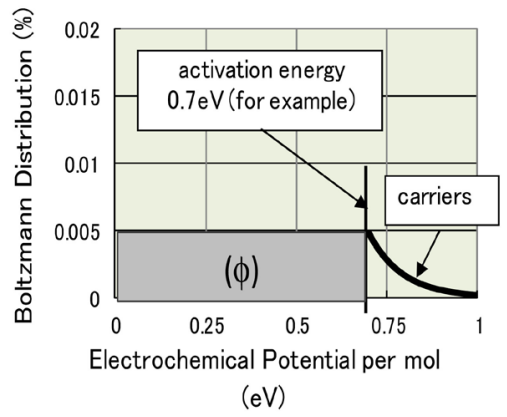

Figure 3. Forbidden distribution of hopping ions.

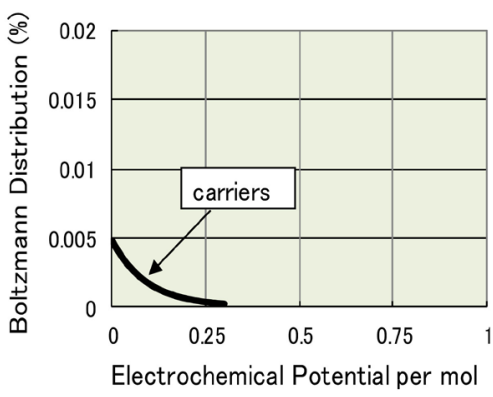

$(\mathrm{eV})$

Figure 4. Correct distribution of hopping ions. 
Ions with energies exceeding the ionic activation energy are converted into charge carriers (i.e., hopping ions).

According to Jarzynski, an accurate distribution should be a canonical ensemble, and the solvation Hamiltonian of mean force $(\phi)$ can be defined [3].

The shape of the distribution in this figure should be the same as the shape of the distribution in Figure 1.

The electrochemical potential can be separated into the chemical potential and the electrostatic potential, as given by

$$
\eta_{i}=\mu_{i}+z_{i} F \varphi
$$

where $z_{i} \eta_{i}, \mu_{i}$ and $\varphi$ are the valence of species $i$, the electrochemical potential, the chemical potential and the electrostatic potential, respectively. In Equation (26), the following transformation should be considered during ion hopping:

$$
\begin{gathered}
\mu_{i \_ \text {hopping }}=\mu_{i_{-} \text {vacancies }}+N E_{a} \\
z_{i} F \varphi_{\text {hopping }}=z_{i} F \varphi_{\text {vacancies }}-N E_{a}
\end{gathered}
$$

where $N, \mu_{i \_ \text {hopping, }}, \mu_{i \text { vacancies }}, \varphi_{i \text { hopping, }}$ and $\varphi_{i_{-} \text {vacancies }}$ are Avogadro's number, the chemical potential of hopping ions, the chemical potential of ions in vacancies, the electrical potential of hopping ions, and the electrical potential of ions in vacancies, respectively. For example, $E_{a}$ is $0.7 \mathrm{eV}$ in SDC electrolytes,

$$
\varphi_{\text {hopping }}=\varphi_{\text {vacancies }}+\frac{E_{a}}{2 e}=\varphi_{\text {vacancies }}+0.35 \mathrm{~V}
$$

When there are enough electrons from the anode side in the SDC electrolyte, due to microscopic interactions, this voltage $(0.35 \mathrm{~V})$ should be neutralized, and the electrical potential energy of ions should be dissipated by electrons. When the Nernst voltage at $1073 \mathrm{~K}$ is $1.15 \mathrm{~V}$, the measured open circuit voltage (OCV) at $1073 \mathrm{~K}$ should be $0.80 \mathrm{~V}(=1.15 \mathrm{~V}-0.35 \mathrm{~V})$. Strictly speaking, the voltage loss can be calculated as follows,

$$
\begin{gathered}
\mathrm{OCV}_{\text {loss }}=\frac{E_{a}}{2 e} \times\left(1-t_{\text {ion }}\right) \\
t_{\text {ion }}=\frac{\sigma_{\text {ion }}}{\sigma_{\text {el }}+\sigma_{\text {ion }}}
\end{gathered}
$$

where $\mathrm{OCV}_{\text {loss }}$ and $t_{\text {ion }}$ are the voltage loss in the OCV and the ionic transference number, respectively. We conclude that the solvation Hamiltonian of mean force $(\phi)$ should be

$$
\phi=N E_{a} .
$$

Therefore, from Equation (27) and Equation (28),

$$
\begin{gathered}
\mu_{i \_ \text {hopping }}=\mu_{i_{-} \text {vacancies }}+\phi \\
z_{i} F \varphi_{\text {hopping }}=z_{i} F \varphi_{\text {vacancies }}-\phi
\end{gathered}
$$

From Equation (30),

$$
\Delta E_{s}=\phi \times\left(1-t_{\text {ion }}\right)
$$

where $\Delta E_{s}$ is the fixed entropy energy difference. Equation (33), Equation (34) 
and Equation (35) demonstrate the central concept of "the modified Wagner's equation".

\subsection{Consideration of the Compatibility with the Jarzynski's Equality}

The compatibility between the Jarzynski's equality and the modified Wagner's equation is discussed in this section. According to Jarzynski [3], the Gibbs volume entropy is given by

$$
W \geq \frac{3}{2} N k T\left[\left(\frac{V_{A}}{V_{B}}\right)^{\frac{2}{3}}-1\right] \geq N k T \ln \left(\frac{V_{A}}{V_{B}}\right) .
$$

In the area of solid-state ionics, the Nernst equation is

$$
\mathrm{OCV}_{\mathrm{th}}=\frac{N k T}{4 F} \ln \left(\frac{p O_{2}^{A}}{p O_{2}^{B}}\right)
$$

where $\mathrm{OCV}_{\text {th }}$ is the Nernst voltage. Similar to Equation (36) and Equation (37), the study on the OCV in the solid-state ionics is related with the Gibbs volume entropy.

The nonequilibrium work relation is described by Jarzynski's equality [3].

$$
\left\langle\mathrm{e}^{-\beta W}\right\rangle=\mathrm{e}^{-\beta \Delta F_{T}}
$$

where $\beta, W$ and $\Delta F_{T}$ are $1 / k T$, the work and the difference in the Helmholtz free energy, respectively. $k$ is the Boltzmann constant. The angular brackets <-> indicate an ensemble over the realizations of the process.

For adiabatic processes, $\Delta F_{T}$ is zero. The following equation should be added [3]:

$$
\left\langle\mathrm{e}^{-\beta X}\right\rangle=1
$$

where $X$ is the energy for the same adiabatic process and ensemble of realizations. In the area of solid-state ionics, $X$ is the ionic activation energy. From Equation (39), an accurate distribution of hopping ions in the electrolytes should be a canonical ensemble. Thus, the Boltzmann distribution during the ion hopping shown in Figure 4 can be explained by the Jarzynski's equality.

Furthermore, the solvation Hamiltonian of mean force $(\phi)$ and the fixed entropy energy difference $\left(\Delta E_{s}\right)$ in Equation (39) can be defined mathematically [3]. Their mathematical approach is too general. In the area of the generalized second law, the separation of the Boltzmann distribution is called "Maxwell's demon".

Consequently, the concept of "the modified Wagner's equation" can be the specialized version of the Jarzynski's equality.

\subsection{Explanation of Our Empirical Equation Using the Modified Wagner's Equation}

\subsubsection{Assumption from Our Empirical Equation}

The explanation of our empirical equation using the modified Wagner's equa- 
tion is discussed in this section for when $m_{p 2}$ is near $m_{p 1}$. We rewrite Equation (1) and Equation (24) again.

$$
\begin{gathered}
\frac{G m_{p}}{r_{p}} \times 1 \mathrm{~kg}=3 k T \\
m_{p 1} c^{2} \times \ln \left(\frac{m_{p 2}}{m_{p 1}}\right)=1 \mathrm{~kg} \times G \times \frac{m_{p 1}}{r_{p 1}}
\end{gathered}
$$

Then, by comparing Equation (40) to Equation (41), we proposed the following equation.

$$
m_{p 1} c^{2} \times \ln \left(\frac{m_{p 2}}{m_{p 1}}\right)=3 k T
$$

When $m_{p 2}$ is near $m_{p 1}$,

$$
\ln \left(\frac{m_{p 2}}{m_{p 1}}\right)=\frac{m_{p 2}-m_{p 1}}{m_{p 1}}
$$

From Equation (42) and Equation (43),

$$
\left(m_{p 2}-m_{p 1}\right) c^{2}=3 k T
$$

Then, we consider the following a pair production.

$$
2 h v \rightarrow p+\bar{p}
$$

where $h, v, p$ and $\bar{p}$ are the Planck constant, frequency, proton and antiproton, respectively. $h v$ does not include both the rest mass and the gravitational potential energy. However, the protons have both the rest mass and the negative gravitational potential energy. Then, we proposed the following equation.

$$
\begin{aligned}
m_{p} c^{2} & =h v-3 k T \\
\phi & =3 k T .
\end{aligned}
$$

Equation (46) and Equation (47) are hinted from Equation (32) and Equation (34). The electrical potential energy (internal energy) of hopping ions should be smaller than that of ions in vacancies. The value of the decrease is equal to the solvation Hamiltonian of mean force $(\phi)$.

This means that the rest mass should decrease from $h v .3 k T(T=3.20 \mathrm{~K})$ is $0.0083 \mathrm{eV}$, which is much smaller than the energy of the rest mass of protons $(938 \mathrm{MeV})$. The increasing ratio is $8.8 \times 10^{-13}$, and the decrease in the rest mass cannot be observed easily.

\subsubsection{Consideration for the Temperature of the Proton}

The solvation Hamiltonian of mean force $(\phi)$ is rewritten.

$$
\phi=3 k T \text {. }
$$

Here, $\mathrm{T}$ is $3.2 \mathrm{~K}$, which is similar to the temperature of the cosmic microwave background of $2.73 \mathrm{~K}$. This does not mean that the temperature of the proton should be near $9 \mathrm{~K}$. 
For example, in the area of solid-state ionics, the solvation Hamiltonian of mean force $(\phi)$ should be equal to the ionic activation energy. When the ionic activation energy is $1.0 \mathrm{eV}$, the calculated temperature is $1.16 \times 10^{4} \mathrm{~K}$. This temperature is different from the background temperature of $1073 \mathrm{~K} .3 \mathrm{kT}$ is the interaction energy between the proton and the rest mass.

The proton consists of three quarks. Thus, the solvation Hamiltonian of mean force $(\phi)$ of one quark is

$$
\phi_{\text {quark }}=k T \text {. }
$$

Equation (49) is more reasonable than Equation (48). The temperature of the cosmic microwave background of $2.73 \mathrm{~K}$ is the universal temperature. The origin of this temperature may be from the interaction energy between the quark and gravity.

\subsubsection{The Lower Limit of the Rest Mass}

The particle, which has a total energy less than $0.0083 \mathrm{eV}(3 \mathrm{kT})$, cannot be influenced by gravity. For example, in the area of solid-state ionics, ions that have an energy less than the ionic activation energy cannot be carriers. The lower limit of the small mass is $0.0083 \mathrm{eV}(3 \mathrm{kT})$. The mass of a neutrino is assumed to be less than $0.5 \mathrm{eV}$. This may provide a solution for the neutrino oscillation problem.

From Equation (35), inevitable dissipation was predicted. When the transference of gravity is not exactly 1 , there should be inevitable dissipation. The interaction coefficient should be on the order of $8.8 \times 10^{-13}$. This value is similar to the value of the weak interaction.

\subsubsection{Consideration for Einstein's Equivalence Principle}

Equation (46) is rewritten.

$$
m_{p} c^{2}=h v-3 k T
$$

In Equation (50), the value of $m_{p 1}$ can be changed. Then, by measuring the decreasing ratio of the rest mass of the proton after a pair is produced in the falling elevator, we can measure the gravitation potential in the elevator. However, according to Einstein, not everybody cannot do it. To solve this problem, we proposed the following equation.

$$
\frac{m_{p 1}}{m_{p 2}}=\frac{k_{1}}{k_{2}}
$$

where $k_{1}$ and $k_{2}$ are the Boltzmann constant in place 1 and in place 2. The Boltzmann constant was defined from the mass of a proton. Thus, Equation (51) is correct. Equation (50) should be

$$
h v=m_{p 1} c^{2}+3 k_{1} T
$$

Then, by using $k_{1} T$ instead of $k T$, in the following Equation (53), the calculated value was constant. Thus, we cannot measure the gravitation potential in the elevator. This means that $3 k T$ should increase with increasing $m_{p}$. Conse- 
quently, the solvation Hamiltonian mean force $(\phi)$ should increase with increasing distance.

$$
\frac{3 k_{1} T}{m_{p 1} c^{2}}=8.8 \times 10^{-13}
$$

\subsubsection{Consideration When $m_{p 2}$ Is Not near $m_{p 1}$}

When $m_{p 2}$ is not near $m_{p 1}$, the explanation for our empirical equation using the modified Wagner's equation is discussed in this section. Equation (42) is rewritten.

$$
m_{p 1} c^{2} \times \ln \left(\frac{m_{p 2}}{m_{p 1}}\right)=3 k T
$$

Thus,

$$
\ln \left(\frac{m_{p 2}}{m_{p 1}}\right)=\frac{m_{p 1} c^{2}}{3 k T}
$$

Thus,

$$
\frac{m_{p 2}}{m_{p 1}}=\mathrm{e}^{\frac{m_{p 1} c^{2}}{3 k T}}
$$

Then, $k$ is a function of the mass of a proton $\left(m_{p}\right)$ and cannot be constant. In Equation (56), the relationship between the rest mass and the distance cannot be obtained. It is impossible to progress any further. This conclusion is the same as the conclusion proposed by Yasuo Katayama in Equation (16).

Using Ted Jacobson's theory, this problem can be solved.

\section{Explanation for Our Model for Gravity}

\subsection{Ted Jacobson's Theory}

Ted Jacobson discovered that the general relative theory is governed by the following Equation (57).

$$
\mathrm{d} S=\frac{\partial S}{\partial E} \mathrm{~d} E+\frac{\partial S}{\partial V} \mathrm{~d} V
$$

where $S, E$ and $V$ are the entropy, the internal energy and the volume, respectively.

\subsection{Our Model for Gravity}

Equation (46) is rewritten.

$$
m_{p} c^{2}=h v-3 k T
$$

In Equation (58), internal energy $(E)$ should decrease. However, the entropy should not decrease. From Equation (57), the Gibbs volume entropy ( $\left.S_{\text {Gibbs }}\right)$ can be defined.

$$
S_{\text {Gibbs }}\left(m_{p} c^{2}\right) \times T=S_{\text {Gibbs }}(h v) \times T+3 k T
$$


Then, we must consider the degree of freedom ( $x, y$ and $z$ direction). In Equation (59), the Gibbs volume entropy ( $\left.S_{\text {Gibbs }}\right)$ for one direction is

$$
S_{\text {Gibbs }}\left(m_{p} c^{2}\right)=S_{\text {Gibbs }}(h v)+k T
$$

Next, $\mathrm{d} V$ in the distance $(r)$ from the mass should be

$$
\mathrm{d} V=4 \pi r^{2} \times \mathrm{d} r
$$

Thus,

$$
\frac{\partial S_{\text {Gibbs }}}{\partial V} \mathrm{~d} V=\frac{S_{\text {Gibbs }}}{\frac{4}{3} \pi r^{3}} \times 4 \pi r^{2} \times \mathrm{d} r=\frac{3 S_{\text {Gibbs }}}{r} \mathrm{~d} r
$$

Therefore, from Equation (60) and Equation (61),

$$
\frac{\mathrm{d} S_{\text {Gibbs }}}{\mathrm{d} r} T=\frac{3 k T}{r}
$$

When the number of protons is $n$,

$$
\frac{\mathrm{d} S_{\text {Gibbs }}}{\mathrm{d} r} T=\frac{3 n k T}{r}
$$

It is important that the direction is opposite to that of the gravitational potential. However, Equation (63) is similar to Newton's Law. $k$ is one of the "defining constants" and becomes part of the 2019 redefinition of SI base units. However, from Equation (53), the value of $k$ should be changed. Equation (53) is rewritten.

$$
\frac{3 k_{1} T}{m_{p 1} c^{2}}=8.8 \times 10^{-13}
$$

It is possible that the increase in the rest mass can be observed on the galaxy scale. Then, $k$ should increase with increasing the mass of a proton. This may provide a solution for the dark matter problem.

\subsection{Consideration for the Origin of the Solvation Hamiltonian of Mean Force $(\phi)$ in the Universe}

The solvation Hamiltonian mean force $(\phi)$ is assumed to originate from the asymmetry of space-time. The asymmetry of space-time was already discovered and is known as "chirality". According to Einstein,

$$
x^{2}+y^{2}+z^{2}-c t^{2}=0
$$

We proposed the following equation.

$$
Z_{1}^{2}+Z_{2}^{2}+Z_{3}^{2}+Z_{0}^{2}=0
$$

where $Z_{1}, Z_{2}, Z_{3}$ and $Z_{0}$ are complex numbers. Then, the asymmetry $\mathrm{e}^{i \theta}$ can be defined. In the area of solid-state ionics, the asymmetry can be produced artificially. For example, YSZ (yttria stabilized zirconia) can be obtained from $\mathrm{ZrO}_{2}$ with an addition of $8 \% \mathrm{Y}_{2} \mathrm{O}_{3}$. Then, ions can move in the $\mathrm{YSZ}$ electrolytes due to the existence of vacancies. Therefore, the ionic activation energy $\left(E_{a}\right)$ can be defined for the ionic conduction. We noticed that the ionic activation energy can be defined as the solvation Hamiltonian mean force $(\phi)$. Then, the Gibbs volume 
entropy can be measured experimentally. Consequently, we proposed the fifth dimension, which is the internal dimension originating from the asymmetry that acts to separate the Boltzmann distribution.

\section{Conclusion}

We discovered an empirical relationship. Then, we examined Yasuo Katayama's theory. Previously, we developed "the modified Wagner's concept" from our own experimental results. We attempted to explain this concept from the generalized second law. The concept of "the modified Wagner's equation" can be the specialized version of the Jarzynski's equality. We examined Ted Jacobson's theory, which is very difficult. However, the basic concept is very easy for us. The Gibbs volume entropy can be defined. Using the Gibbs volume entropy, we tried to explain our empirical equation and tried to comprehensively develop Yasuo Katayama's theory. The calculated temperature was $3.20 \mathrm{~K}$, which is similar to the temperature of the cosmic microwave background of $2.73 \mathrm{~K}$ and was much larger than the Unruh temperature. Several topics were suggested for future discussion.

\section{Conflicts of Interest}

The author declares no conflicts of interest regarding the publication of this paper.

\section{References}

[1] Jacobson, T. (1995) Physical Review Letters, 75, 1260-1263. arXiv:gr-qc/9504004 https://doi.org/10.1103/PhysRevLett.75.1260

[2] Verlinde, E.P. (2011) Journal of High Energy Physics, 2011, Article Number: 29. arXiv: 1001.0785 https://doi.org/10.1007/JHEP04(2011)029

[3] Jarzynski, C. (2017) Physical Review X, 7, 011008. https://doi.org/10.1103/PhysRevX.7.011008

[4] Miyashita, T. (2020) Open-Circuit Voltage Anomalies in Yttria-Stabilized Zirconia and Samaria-Doped Ceria Bilayered Electrolytes. https://ecsarxiv.org/xhn73/ https://doi.org/10.1149/osf.io/xhn73

[5] Katayama, Y. http://home.catv.ne.jp/dd/pub/pot/pot.html\#0

[6] Miyashita, T. (2006) Journal of Materials Science, 41, 3183. https://doi.org/10.1007/s10853-006-6371-8

[7] Miyashita, T. (2011) Electrochemical and Solid State Letters, 14, 1. https://doi.org/10.1149/1.3581208

[8] Miyashita, T. (2017) Journal of the Electrochemical Society, 164, 3190. https://doi.org/10.1149/2.0251711jes 